\title{
ARRAYS OF CRICKET-INSPIRED SENSORY HAIRS With Capacitive Motion Detection
}

\author{
J. van Baar, M. Dijkstra, R. Wiegerink, T. Lammerink, R. de Boer and G. Krijnen \\ $\mathrm{MESA}^{+}$Research Institute, University of Twente \\ P.O. Box 217, 7500 AE Enschede, The Netherlands
}

\begin{abstract}
This paper presents the fabrication of flow-sensors based on the drag-force induced motion of artificial hairs connected to capacitive read-out. Artificial hairs were made either out of moulded silicon-nitride structures or by SU-8. The SU-8 hairs were suspended on membranes containing electrodes to form the variable capacitors. Silicon-rich-nitride hairs were made using a silicon wafer as a dissolvable mould. The longest SU-8 hairs were fabricated using a $470 \mu \mathrm{m}$ thick photoresist layer. Capacitance versus voltage, frequency dependency and directional sensitivity measurements have been carried out on entire arrays and are reported in this paper.
\end{abstract}

\section{INTRODUCTION}

Flow velocity can be measured in various ways, e.g. thermally using a hot wire [1], by measuring the pressure drop in a channel [2] and by measuring the drag force on an obstacle [3]. The latter is used for the artificial hair sensors presented in this paper, which are based on the flow-sensing hairs on the cerci of a cricket, see Fig. 1. Crickets have hairs to sense approaching predators. The hairs have variable length and diameter, the distribution being bimodal with concentrations around 150 and 750 micron for adults [4].

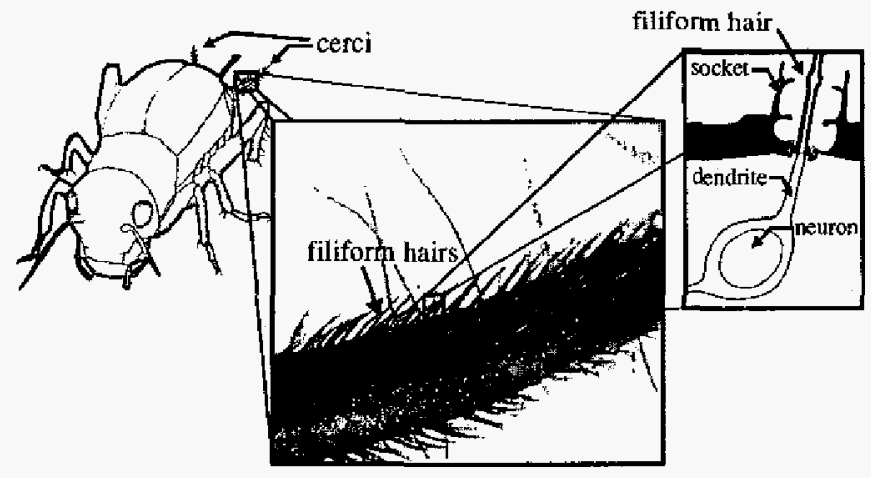

Figure 1: Flow-sensitive hairs on the cerci of a cricket.

Several groups have been working on the realization of artificial hairs for flow sensing $[5,6]$. Two basic types of artificial hairs can be distinguished, namely hairs fabricated in the wafer plane and hairs fabricated perpendicular to the wafer plane. Fabrication of hairs in the wafer plane is most straightforward since surface micromachining techniques can be used. However, although the fabrication process is straightforward, surface micromachined hairs are of limited use because they cannot easily be combined into larger arrays. Chen, et al. proposed to use the so-called plastic deformation magnetic assembly (PDMA) method to erect the hairs out of the wafer plane [6]. In the PDMA process, a magnetic field is used to bend surface micromachined beams. The beams are plastically deformed, so that they remain bent after the magnetic field is removed.

Our research has been focused on fabrication of hairs perpendicular to the wafer surface. Two fabrication processes have been investigated. In the first process, a silicon wafer is used as a mould to realize silicon-richnitride ( $\mathrm{SiRN}$ ) hairs. In this way a high density of hairs can be realized. The second fabrication process uses hairs of SU-8 photoresist. These hairs can be realized on top of a surface-micromachined read-out structure.

\section{MODEL}

Fig. 2. shows a schematic drawing of a gimbal-suspended sensory hair, rotating around one axis with capacitive read-out. The structure can be described by a three-port transducer model: two mechanical ports for rotation and translation of the structure and a third, electrical, port for the parallel plate capacitor. The parallel plate capacitors are formed by the electrodes on the movable plate and by a counter electrode below the moveable plate.

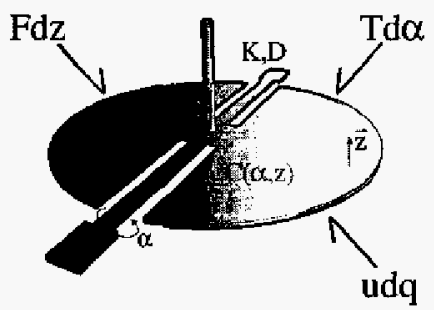

Figure 2: Three-port transducer model describing artificial sensory hairs.

The total derivative of the energy for this transducer is given by:

$$
d E=T d \alpha+F d z+u d q
$$

Here $T$ is the external torque, $F$ the extemal force (in $\mathrm{z}$ direction and $u$ the voltage over the capacitor. The energy is obtained by partial integration of Eq. 1., using the fact that $q=\mathrm{C}(\alpha, z) \cdot u$. The capacitance depends both on rotation angle and translation.

$$
E(\alpha, z, q)=\int T(\alpha) d \alpha+\int F(z) d z+\int \frac{q}{C(\alpha, z)} d q
$$


Since the structure contains basically only three energy buffers the energy function can be relatively easily obtained provided one assumes linear torsional and translational stiffnesses:

$$
E=\frac{I}{2} K\left(\alpha-\alpha_{0}\right)^{2}+\frac{I}{2} D\left(z-z_{0}\right)^{2}+\frac{q^{2}}{2 C(\alpha, z)}+E_{b}
$$

where $E_{b}$ is a base energy level that is not affected by the transduction process. $K$ accounts for the torsional spring stiffness and $D$ accounts for the translational spring stiffiness. It is assumed that translation, $z$, and rotation, $\alpha$, are independent of each other. Force balance and torque balance equations are obtained by differentiation of Eq. 3 . with respect to $\alpha$ and $z$ :

$$
\begin{aligned}
F=\left(\frac{\partial E}{\partial z}\right)_{\alpha q} & =D\left(z-z_{\theta}\right)-\frac{I}{2} \frac{q^{2}}{C^{2}}\left(\frac{d C}{d z}\right) \\
& =D\left(z-z_{0}\right)-\frac{I}{2}\left(\frac{d C}{d z}\right) u^{2}=0 \\
T=\left(\frac{\partial E}{\partial \alpha}\right)_{z, q} & =K \alpha-\frac{I}{2} \frac{q^{2}}{C^{2}} \frac{\partial C}{\partial \alpha} \\
& =K \alpha-\frac{I}{2} u^{2} \frac{\partial C}{\partial \alpha}=T_{\text {drag }}
\end{aligned}
$$

$\mathbf{T}_{\text {drag }}$ is the drag torque on the hair by the airflow. Since no analytical expression was available for $\mathrm{C}(\alpha, z)$, Eq. 4 . and Eq. 5 . were solved numerically using trapezoid integration over infinitesimal parallel plate subsections. $D$ was calculated from the deflection curve of a beam clamped on both sides, with a point load at the centre of the beam. $K$ was calculated by using Saint-Venant's approximation for a beam under torsion.

Fig. 3. shows the calculated capacitance as a function of constant drag-force on the hair for a gimbal-suspended sensor with a plate diameter of $200 \mu \mathrm{m}$ and a gap distance of $2 \mu \mathrm{m}$ for various values of the applied voltage. The higher the voltage the more sensitive the structure is.

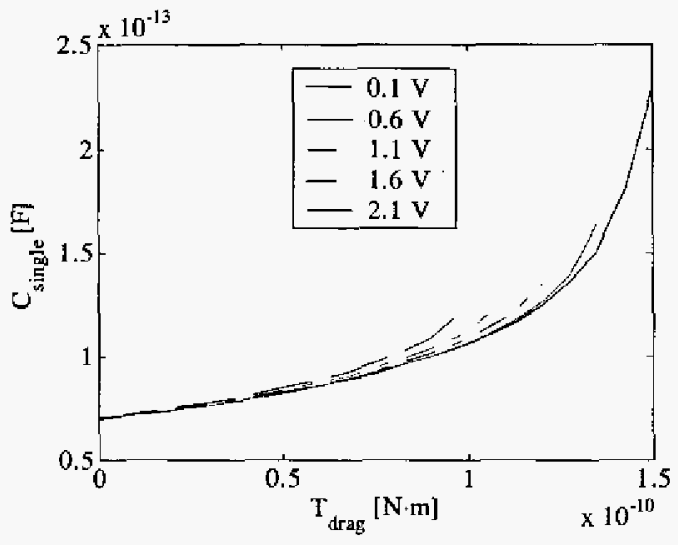

Figure 3: Capacitance as a function of drag-force on the hair, calculated for various voltages.

Fig. 4. shows calculated C-V curves for the same configuration, but without application of a drag force. In one case both electrodes are assumed to be activated, inducing only translation (grey line). In the other case only one side of the capacitor is used resulting in translation and rotation (black line).

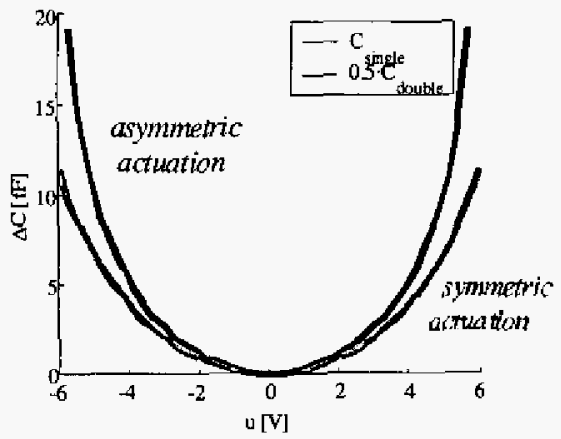

Figure 4: Calculated capacitance change as a function of applied bias voltage.

\section{DESIGN AND FABRICATION}

\section{Silicon-rich-nitride hairs using Si moulds}

Fig. 5. shows a condensed fabrication process flow of SiRN hairs that served to investigate the possibilities to etch hairmoulds in $\mathrm{Si}$ and to determine the allowable aspect ratios. The hairs are made by Deep Reactive Ion Etching (DRIE). To this end, mask openings ranging from 5 up to 50 microns, and various shapes, like circle, square, triangle, ellipse and star, were examined. Hairs with a large diameter have two advantages: it takes relative little time to etch them by DRIE and the drag force is larger due to the larger frontal area. A disadvantage is the large mask opening: large holes in the wafer surface prevent proper spimning of resist and complicate further processing steps.

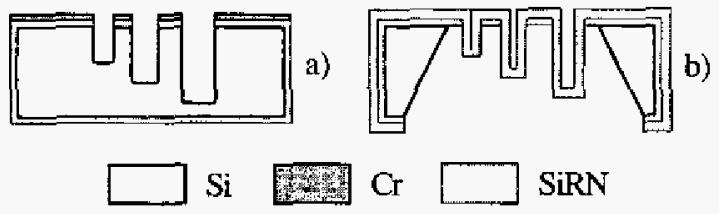

Figure 5: Process sequence of SiRN hairs on a membrane.

Processing starts with the deposition of a $20 \mathrm{~nm}$ thin SiRN layer by low-pressure chemical vapor deposition (LPCVD) on a $0.5 \mathrm{~mm}$ bare $\langle 100\rangle$ silicon wafer as an adhesion layer for a $20 \mathrm{~nm}$ sputtered chromium layer. Between lithography and etching of the chromium layer an ozone step is required for sufficient wetting of the small pores; otherwise the chromium will not etched. Next, the deep holes are realized by DRIE. These holes are then filled by a $1 \mu \mathrm{m}$ thick low-stress LPCVD silicon-rich-nitride layer. Finally, the silicon bulk is etched away from the backside using $\mathrm{KOH}$ so that the hairs on the membrane are released.

From the SEM photographs in Fig. 6. it can be seen that the cross pattern develops into a circular shape and the star shape into a square. Eventually all mask openings will end up into squares due to the anisotropy of the $<100\rangle \mathrm{Si}$ wafer. 


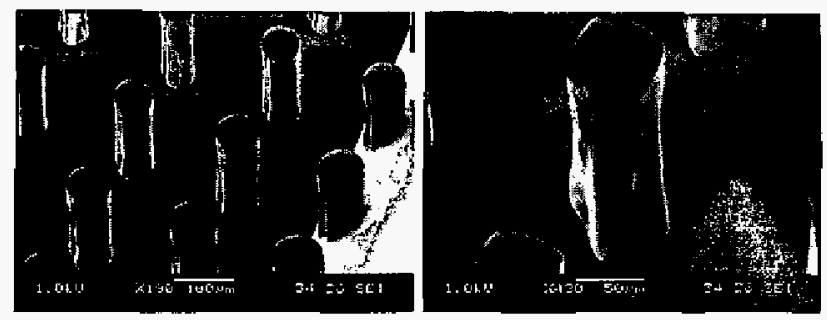

Figure 6: SEM photographs of hairs with a cross or star shaped opening, developing into a rectangular shape.

Due to the RIE lag, depending on hair diameter, hair heights in one run ranged from 100 to 250 micron after 3 hours of DRIE, see Fig. 7 . The flat top and negative tapering indicate that continued etching will result in even longer hairs.
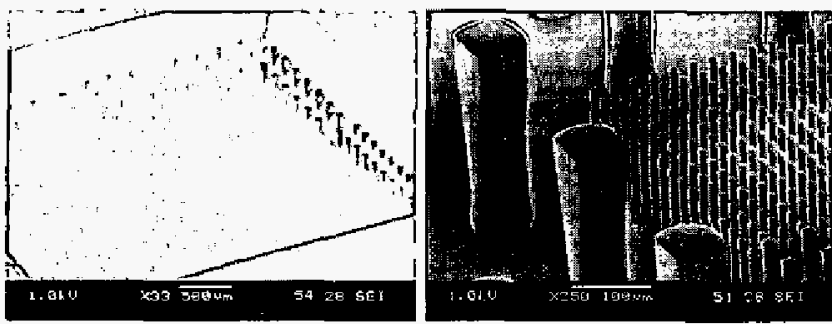

Figure 7: Hairs with varying diameters of the mask openings (5-50 micron) and therefore varying height.

\section{Capacitive sensor structures with SU-8 hairs}

Fig. 8. shows a schematic drawing of a surface micromachined suspended membrane with a hair of SU-8. Two types of suspensions have been designed: spiral and gimbal. The membrane has a diameter that ranges from 50 up to 250 microns and it can rotate around one or two axes. This version of the devices does not have any shielding above the membrane. Therefore these structures may be sensitive to both drag and shear-forces induced by the airflow.

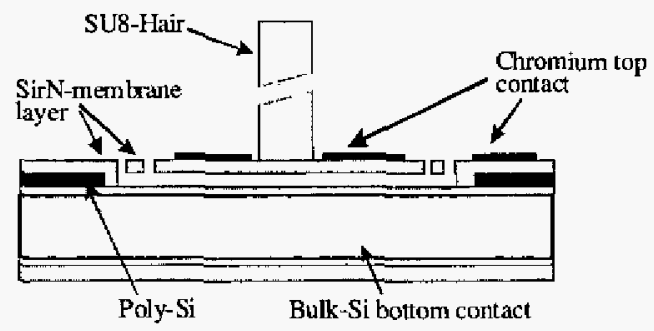

Figure 8: Hairs with varying diameters of the mask openings (5-50 micron) and therefore varying height.

Processing of the complete sensor structures with capacitive read out starts with a highly conductive silicon wafer that is used as one of the electrodes of the capacitor. A thin silicon nitride layer of $100 \mathrm{~nm}$ is deposited as a protection layer for the underlying silicon. A $1 \mu \mathrm{m}$ thick LPCVD poly-silicon layer that defines the gap between the membrane and the substrate is deposited (Fig. 9a.) and patterned (Fig. 9b). Next, a $1 \mu \mathrm{m}$ LPCVD silicon nitride layer is deposited and pattemed which forms the suspension springs and the moveable membrane (Fig. 9c.). Chromium electrodes of $20 \mathrm{~nm}$ are sputtered and patterned on top of the siliconnitride (Fig. 9d.). A thick SU-8 layer is spin-coated on the surface (Fig. 9e). First devices had a layer thickness of $200 \mu \mathrm{m}$ whereas later $470 \mu \mathrm{m}$ has been achieved. Further optimization to over $1 \mathrm{~mm}$ seems possible [7]. Next (Fig. 9f) the SU-8 is illuminated with the hairs pattern and developed. Finally the devices are released by isotropic plasma etching of the poly-silicon sacrificial layer (Fig. 9g).

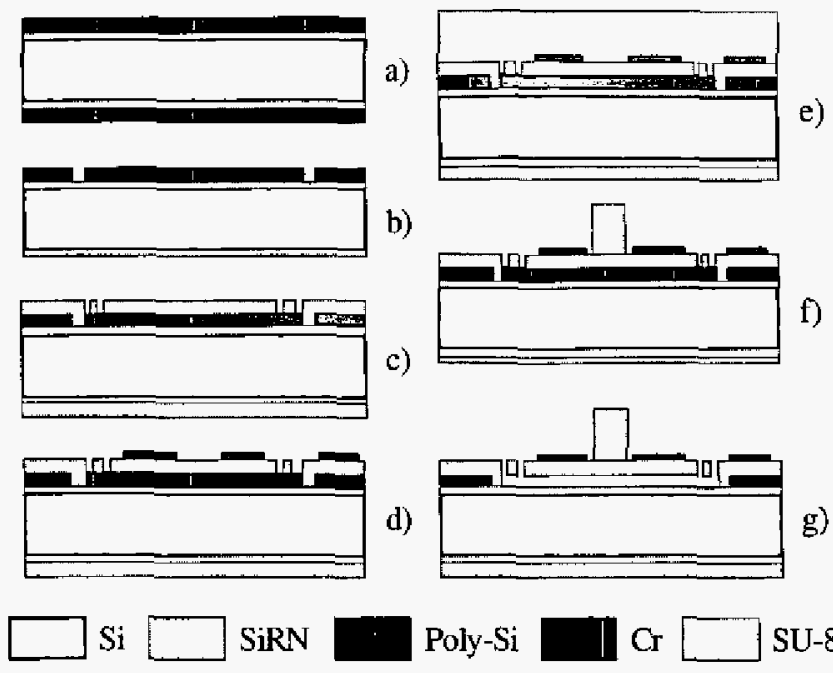

Figure 9: Condensed process flow of $S U-8$ hairs on top of suspended SiRN membrane with chromiam electrodes.

Fig. 10. shows a SEM photograph of an SU-8 hair on top of a suspended membrane with $\mathrm{Cr}$ top electrodes (left) and an array of sensory hairs (right).

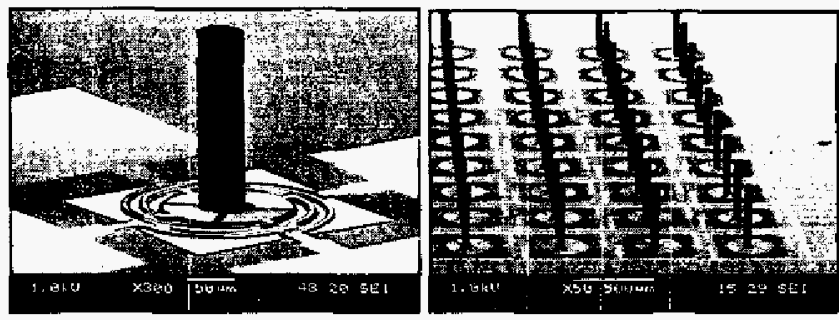

Figure 10: SEM photographs $S U-8$ hair of $200 \mathrm{um}$ height (left) and an array of hairs of $470 \mu \mathrm{m}$ height (right).

\section{Measurements}

Using an HP 4194A impedance analyzer two types of $\mathrm{C}-\mathrm{V}$ measurements were done for electrical characterization of the devices: by application of a bias voltage to all electrodes and a bias voltage to half of the electrodes only. The first gives a symmetric electrostatic force towards the silicon bulk and therefore results in a translation directed downwards. The latter results in both rotation and translation of the device.

Fig. 11. shows C-V measurements where the effect of translational and rotational movements can be observed in the change in capacitance. It can be seen that the capacitance change for gimbal-suspended sensors is slightly higher when only half of the device is actuated. This means that the device translates more easily than it rotates. spiralsuspended sensors show an opposite behavior: the changes in capacitance are higher when the device is actuated completely. 


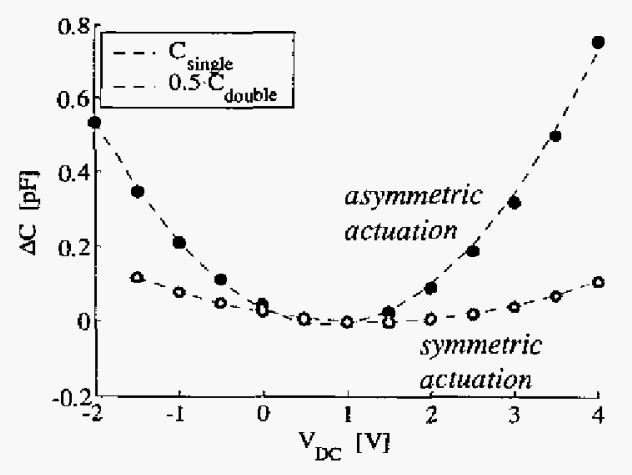

Figure 11: C-V measurements on an array of gimbalsuspended sensory hairs.

Characterization with oscillatory acoustic flows where done using an electrical signal frequency of $1 \mathrm{MHz}$, a charge amplifier and synchronous detection for capacitive measurements (Fig. 12.).

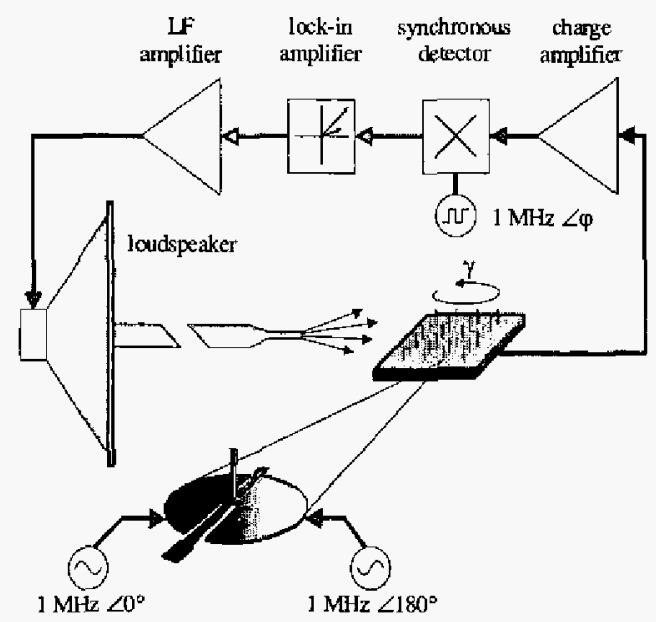

Figure 12: Flow measurement setup.

The characteristics of the flow source, a loudspeaker, have been eliminated by normalization to the particle velocity of the sound wave as measured by a microflown [8]. Resonance of the sensor was not observed, within the measurement range of $100 \mathrm{~Hz}$, limited by the used loudspeakers (Fig. 13.).

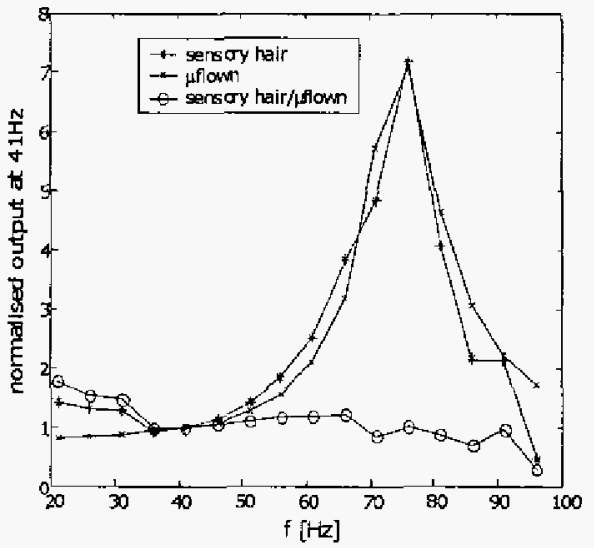

Figure 13: Frequency response of a hair sensor array, $a$ microflown and normalized hair-sensor response.
The directional sensitivity as shown in Fig. 14, displays a figure of eight, showing that the sensor has a preferred oscillating direction. A sharp transition can be observed in the phase at $0^{\circ}$ and $180^{\circ}$, this is where the flow changes sign over the electrodes.

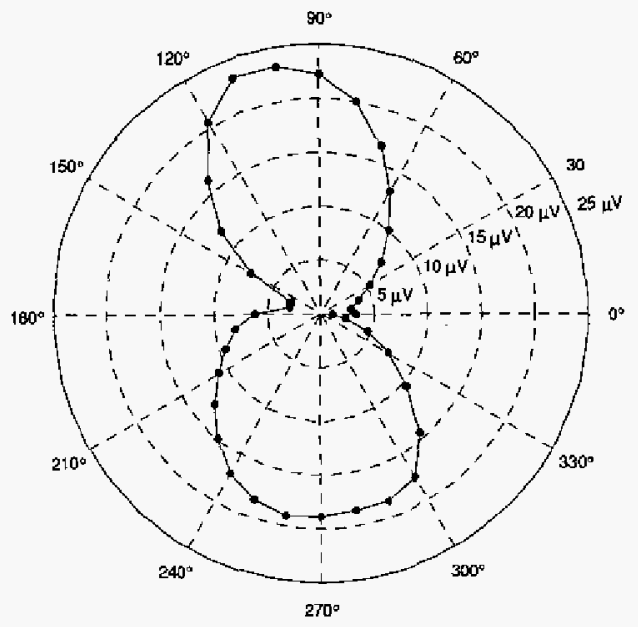

Figure 14: Directivity measurements, amplitude versus $\gamma$

\section{Conclusions}

We have shown two processes for fabrication of arrays of hairs for drag force sensing. The fabrication of hairs on a membrane by filling deep etched holes turned out to be more complicated than fabrication of SU-8 hairs on top of a membrane. Highest fabricated SU-8 hairs were 470 microns, but will be even higher in the future. Directivity measurements show an expected figure of eight. Future research will be aimed at further characterization of the hair-sensors, improved sensitivity and higher connectivity.

\section{REFERENCES}

[1] G.C.M. Meyer and A.W. van Herwaarden, Thermat sensors, Institute of Physics Publishing, ISBN 0-7503-0220-8.

[2] F.P. Incropera and D.P. DeWitt, Fundamentals of Heat and Mass Transfer, John Wilcy 1996.

[3] F.M. White, Fluid Mechanics, McGraw-Hill, 1994

[4] O. Dangles, J. Casas, Institut de Recherche en Biologie de $1^{*}$ Insecte, private communication.

[5] Y. Ozaki, T. Ohyama, T. Yasuda, and I. Shimoyama, "An air flow sensor modeled on wind receptor hairs of insects," Proc. MEMS 2000 (Miyazaki, Japan), pp. 531-537, 2000.

[6] J. Chen, Z. Fan, J. Zou, J. Engel, and C. Liu, "Two dimensional micromachined tlow sensor array for fluid mechanics studies," Journal of Aerospace Engineering, Vol. 16, No. 2, pp. 85-97, 2003.

[7] Lorenz et.al., Sensors \& Actuators A, Vol-64, 1998, p33

[8] H-E. de Bree et al., The Microflown: a novel device measuring acoustical flows, Sensors and Actuators A 54, (1996) $552-557$

\section{ACKNOWLEDGEMENTS}

The authors want to thank: Erwin Berenschot and Meint de Boer for their advice on processing, our colleagues in the EU project CICADA for stimulating discussions and input to this work and the EU for financing support through project IST-2001-34718 (CICADA) in the Lifelike Perception program. 